\title{
Editorial
}

\section{Low and High Radio-Iodine-131 Doses in Postoperative Ablation in Differentiated Thyroid Carcinoma}

\author{
Elrasad, Sh. \\ Nuclear Medicine Unit, NEMROCK Center, Cairo University, Egypt
}

The goals of post-surgical RAI therapy in patients with differentiated DTC are to ablate residual normal thyroid tissue that may facilitate surveillance,) Also the potential tumoricidal effect on residual microscopic RAI-avid disease, and to provide a posttreatment whole-body scan that may reveal undetected local or distant metastases.Although, these goals are important, the ultimate endpoint of postsurgical ablation is to minimize DTC recurrence and death by eliminating residual normal thyroid tissue or residual microscopic disease that could be a focus for future recurrence(1).

Successful RAI-131 thyroid remnant ablation is associated with better prognosis with regard to both recurrence-free and overall survival, lower rates of distant metastases, and reduced cancer mortality rates, compared with only surgery or surgery and L-thyroxin therapy alone (2). It also facilitates long-term follow up of patients with DTC. It was shown by Verburg et al. that a successful ablation itself seems to be a highly important prognostic factor for long-term outcome. They found that the patients with a successful ablation, $87 \%$ were still free of the disease after 10 years, whereas of the patients with an unsuccessful ablation, only $50 \%$ were free of disease with thyroid cancer-related survival was $(93 \%$ versus $78 \%)(\mathrm{P}>0.001)(3)$.

After four decades of follow-up, based on regression modeling of 1510 patients without distant metastases at the time of initial therapy, Mazzaferri and Kloos found thyroid remnant ablation to be an independent variable that reduced loco regional recurrence, distant metastases, and cancer death. A similar observation has also been made by the National Thyroid Cancer Treatment Cooperative Study group (4), they had reconfirmed that postoperative RAI treatment was associated with improved cancer-specific mortality rates and reduced disease progression in both papillary and follicular cancer(5).

Side effects and risks of radioactive iodine therapy have been reported, including salivary 
gland problems (dry mouth, alterations in taste, or salivary gland pain),

Lacrimal problems (watery eyes, and tear duct blockage) (6), risk of dental caries and oral problems (post-RAI xerostomia). Loss of taste that can become permanent) (7), as well as increased risk of secondary primary malignancies from high-dose RAI therapy (8, 9).

All previous data suggests that it is important to achieve complete ablation after diagnosis in order to ensure the best possible prognosis for a patient. And to reduce the ablative RAI131 dose as much as possible to reduce the incidence of the side effects.

ATA guidelines for RAI dose selection is based on patient risk factors. It states that lowrisk patients receive $80-120 \mathrm{mCi}$, according to the risk of patients. The ATA guideline states that patients should receive the least dose that could achieve complete ablation (10).

Regarding administered activities, published guidelines give no strong conclusions on the level of activity to administer for an ablation, which can vary from 30 to $100 \mathrm{mCi}$ for remnant ablation that generally may show similar rates of successful remnant ablation and recurrence rates. However, the goal is to administer the lowest activity possible that will effectively eliminate the residual normal thyroid tissue (10-12).
Many studies done to evaluate the effect of therapeutic dose concluded showed that there is no significant difference regarding the ablation outcome between the high and the low RAI-131in low risk group (2, 12-23).

In patients defined as high risk according to ATA and ETA guidelines (10, 24), high RAI activities are recommended. Much debate still exists regarding the activities administered in intermediate risk (microscopic invasion of tumor into the peri-thyroidal soft tissues, aggressive tumor histology, and node positive). In this context, no evidences are provided regarding the optimal RAI activity to be administered and current recommendations, advocating the use of high RAI activities, are based mainly on the expert opinion rather than clinical evidence $(10,24)$.

\section{Low Dose Ablation:}

Elrasad et al.(2014), a prospective, randomized trial ever performed that involved 88 patients of low and intermediate risk with complete tumor and pathologic LN excision, and no evidence of residual disease in postoperative evaluation by ultrasonography. Patients were randomized into two groups then we prospectively compared the overall response rate in each group. They found that 23 patients in group 1 from a total of 39 (58.9\%) included in Low RAI131-dose group, have achieved successful ablation with no significant residual functioning thyroid tissue 
in the first follow-up DxWBS scan done after 6-9 months, TG level $<2 \mathrm{ng} / \mathrm{ml}$, and no cervical

LN's on follow up US. The response rate in group II was (75.5\%) (37 patients from a total of 49) $(\mathrm{P}$ value $=0.098)$. Although no significant difference in ablation rate between the two groups.However, Limitations of this study were; small number of patients and short follow up duration (6-18 months)(25).

Similar results were reported by Castagn et al. in a retrospective study, involved 225 DTC patients classified as intermediate risk. They stratified DTC patients in two homogenous groups, according to the RAI activity given for remnant ablation: 85/225 patients (37.8\%) were treated with low RAI activities (1110$1850 \mathrm{MBq})$ and 140/225 (62.2\%) with high RAI activities (>3700 MBq). All patients had been treated with near total thyroidectomy; all patients received RAI therapy after thyroid hormone withdrawal (THW) or after recombinant human TSH. At 6-18 months (median 9 months) after initial therapy, patients were evaluated to define the clinical status. When stimulated serum TG was undetectable $(<1.0 \mathrm{ng} / \mathrm{ml})$ and neck US (and diagnostic WBS when performed) were negative, patients were defined as in remission. 56(40\%) patients treated with low and 84/140 (60\%) patients treated with high RAI activities fulfilled the criteria for remission No difference in clinical status was found between patients treated with low or high RAI-131 activities $(\mathrm{P}$ value $=0.56)$. Biochemical disease (detectable serum TGwith no evidence of disease) was found in 16/85 $(18.8 \%)$ patients treated with low and in 20/140 (14.3\%) patients treated with high RAI-131 activities. Metastatic disease was found in 18/85 (21.2\%) patients treated with low and in 36/140 (25.7\%) patients treated with high RAI-131 activities (11).

Also, Mallick et al., 2012 also concluded that low-dose radioiodine plus thyrotropinalfa was as effective as high-dose radioiodine, with a lower rate of adverse events in their randomized study that was conducted at 29 centers in the United Kingdom involving 438 patients comparing low-dose and high-dose radioiodine, each in combination with either thyrotropinalfa or thyroid hormone withdrawal before ablation. Ablation success rates were $85.0 \%$ in the group receiving low-dose radioiodine versus $88.9 \%$ in the group receiving the high dose and $87.1 \%$ in the thyrotropinalfa group versus $86.7 \%$ in the group undergoing thyroid hormone withdrawal. Similar results were found for low-dose radioiodine plus thyrotropinalfa $(84.3 \%)$ versus high-dose radioiodine plus thyroid hormone withdrawal $(87.6 \%)$ or highdose radioiodine plus thyrotropinalfa $(90.2 \%)$. They suggested that high-dose group needs patient hospitalization for at least 3 days (36.3\% vs. $13.0 \%, \mathrm{P}<0.001)$. The proportion of patients with adverse events were $21 \%$ in 
the low-dose group versus $33 \%$ in the highdose group $(\mathrm{P}=0.007)$ and $23 \%$ in the thyrotropin alfa group versus $30 \%$ in the group undergoing thyroid hormone withdrawal $(\mathrm{P}=0.11)$ (12).

In the study of Schlumberger, et al,(2012) they stated that the use of recombinant human thyrotropin and low-dose $30 \quad \mathrm{mCi}$ postoperative RAI-131 ablation may be sufficient for the management of low-risk thyroid cancer. It was randomized trial of 715 patients, comparing $30 \mathrm{mCi}$ versus $100 \mathrm{mCi}$ Inclusion criteria were an age of 18 years or older; total thyroidectomy for DTC; absence of distant metastasis; and no iodine contamination. Thyroid ablation was assessed 8 months after radioiodine administration by neck ultrasonography and measurement of recombinant human thyrotropin-stimulated thyroglobulin. Ultrasonography of the neck was normal in $652(95 \%)$, and the stimulated thyroglobulin level was $1.0 \mathrm{ng}$ per milliliter or less in 621 of the 652 patients $(95 \%)$ without detectable thyroglobulin antibodies. Thyroid ablation was complete in 631 of the 684 patients $(92 \%)$. The ablation rate was equivalent between both ${ }^{131}$ I doses (23).

Furthermore,Bal, et al. 2012, reported that first-dose ablation rates at 6 months in the stratified randomized trial conducted on 450 patients to compare between lower RAI-131 activities (25 and $50 \mathrm{mCi}$ ) and activities as high as $100 \mathrm{mCi}$. All patients underwent pre- ablation RAI-131 whole-body scan, 48-h radioiodine neck uptake measurements and post-therapeutic scans. Evaluation was performed after 6 months. First-dose ablation success rate was $81.5,84.9,88.5$, and $84.2 \%$ in the 25,50 , and $100 \mathrm{mCi}$ groups and overall, respectively. Histology had no effect on ablation rate (16).

Another study of Caglar, et al.(2012), suggested that remnant thyroid tissue in patients with low-risk, well-differentiated thyroid cancer after total thyroidectomy can be ablated with $21 \mathrm{mCi}$ of I-131. The success rate is not different from that obtained with $100 \mathrm{mCi}$ I-131. It was conducted on a total of 108 non metastatic low-risk patients with DTC had I-131 ablation for the postoperative thyroid remnant. 53 patients received a low dose $21 \mathrm{mCi}(800 \mathrm{MBq})$ and 55 patients received a high dose $100 \mathrm{mCi}(3700 \mathrm{MBq})$ of I-131 after total thyroidectomy. In low dose RAI group, 32 out of $53(60 \%)$ and 43 out of $53(81 \%)$ patients were successfully treated versus 35 out of $55(64 \%)$ and 42 out of 55 (76\%) for high dose RAI group, respectively $(\mathrm{P}=\mathrm{NS})$. The differences were not statistically significant between the two groups (17).

\section{High Dose Ablation:}

On the other hand Prpic, et al.( 2012), evaluated 259 DTC patients confined to the thyroid has reported that $100 \mathrm{mCi}$ of RAI was significantly more effective in thyroid ablation 
than $30-50 \mathrm{mCi}$ and also superior to $75 \mathrm{mCi}$. However, ablation rates were nearly similar after a second RAI dose. This second dose ranged from 30-100 $\mathrm{mCi}$. Unfortunately, the authors did not explain their rationale for the second ablation dosage, plus the results of the first ablative dose activity outcome was contradictory to that of the second dose(26).

Also a meta-analysis of 19 studies by Doi\& Woodhouse on 2000 compared 518 patients ablated with $30 \mathrm{mCi}$ against 449 ablated with $100 \mathrm{mCi}$, they found that $100 \mathrm{mCi}$ of RAI was significantly more effective and concluded that one in seven recurrences would have been prevented by high-dose ablation(27).

Furthermore Pacini, et al. stated that 'when using stimulation with rhTSH, a $30 \mathrm{mCi}$ standard dose of RAI is not sufficient for satisfactory thyroid ablation' (28).

\section{No RAI ablation in low- or intermediate- risk DTC patients:}

Rosario et al. (2012) in their study of 136 Patients who had initial stimulated TG values of $<1.0 \mathrm{ng} / \mathrm{ml}$, and their follow-up consisted of monitoring non-stimulated serum TG levels and neck ultrasound. None of these patients had the further assurance of 'absent disease' with a negative post-RxWBS. Lymph node metastases were 'excluded' by ultrasound and the lack of suspicious nodes at surgery. Prophylactic cervical node dissection was not performed. Patient follow-up ranged from 12 to 72 months (mean, 44). They concluded that a larger number of patients with longer followup were needed to confirm this approach in the post-operative follow-up of 'low-risk' nonRAI-ablated DTC patients (29).

AlsoIbrahimpasic, et al,(2012), recommended no post-operative RAI ablation in low and intermediate-risk patients with un-stimulated $\mathrm{TG}<1.0 \mathrm{ng} / \mathrm{ml}$, claiming equivalent outcome when comparing non-RAI-treated patients with RAI-treated patients. The authors support using un-stimulated TG, '.because recurrence rates are so low with un-stimulated TG measurements, one can argue that the routine use of stimulated $\mathrm{TG}$ in these patients. The limitations of this follow up methodology using stimulated TGfor detecting DTC recurrence have been described earlier. The mean patient follow up was a relatively short duration of 59 months. Careful scrutiny also reveals that this article's cohorts were not equivalent, and there were two major differences; out of 75 patients, 69 (92\%) with known nodal metastasis received RAI ablation, while $6(8 \%)$ were not ablated and among the intermediate-risk patients, based on the authors' classification system, more than $90 \%$ (73/81) of patients were treated with RAI ablation while $<10 \%(8 / 81)$ were not. This indicates that the results are skewed against RAI ablation as the RAI-ablated cohort was at a higher risk than the cohort without ablation. $(30,31)$. 
Another publication in 2013 has reported that the number of metastatic DTC nodes should be determined before post-operative RAI ablation is performed (Tuttle \& Sabra)(32). they recommended no RAI with nodal metastasis unless there are more than ten metastatic nodes that are $<5 \mathrm{~mm}$ in size, or more than five metastatic nodes of which the majority are 5$15 \mathrm{~mm}$ in size, or any single node more than $15 \mathrm{~mm}$ in size. The authors state, '.these are clearly arbitrary criteria for indications of RAI treatment. Accordingly, the validity of this approach is unestablished, stressing the need for prospective trials that would confirm or refute these criteria and in order to firmly establish the clinical management that would minimize the risk of recurrent DTC(32).

\section{Follow up and Risk of Recurrence Following RAI Ablation:}

An obvious concern before adopting low-dose iodine ablation is its effect on disease recurrence on follow up.Kukulska et al. in 2010 concluded that no significant differences in the 5 years efficacy of thyroid remnant radioiodine ablation using 30, 60 and $100 \mathrm{mCi}$ were observed in low-risk DTC patients operated by total thyroidectomy and neck lymph node dissection. The study involved 309 DTC patients with no clinical, histopathologic, sonographic or biochemical signs of persistent disease after total thyroidectomy and appropriate extent of neck lymph node dissection. For radioiodine thyroid remnant ablation, $30 \mathrm{mCi}$ of ${ }^{131} \mathrm{I}$ was applied in 86 patients, whereas $60 \mathrm{mCi}$ in 128 and 100 $\mathrm{mCi}$ in 95 patients. The median follow-up was 10 years for subjects treated with $30 \mathrm{mCi}$ and $60 \mathrm{mCi}$ and 6 years for patients treated with $100 \mathrm{mCi}$ of ${ }^{131} \mathrm{I}$. After first dose ablation, a second ${ }^{131}$ I treatment was necessary in $10 \%$ patients, without difference between groups treated with 60 and $100 \mathrm{mCi}$ and in $22 \%$ patients treated with $30 \mathrm{mCi}$. All patients entered full remission. To evaluate the longterm outcome of the adjuvant ${ }^{131} \mathrm{I}$ treatment, the course of the follow-up and the most recent disease status were assessed by sonography, radiological examinations and serum $\mathrm{TG}$ estimation (on LT4- suppressive treatment). Within the whole observation period local relapse was stated in $2(2.4 \%), 4(3 \%)$ and 3 (3\%) patients treated with ${ }^{131} \mathrm{I}$ activities of 30 $\mathrm{mCi}, 60 \mathrm{mCi}$ and $100 \mathrm{mCi}$ respectively and serum TG concentration on LT4-suppressive treatment was low, without differences between groups(21).

Another study of Kuruijff, et al., (2013) reported that decreasing the dose of RAI at initial ablation for patients with pT1-pT3 PTC does not seem to be associated with an increased risk of structural cancer recurrence. They conducted a retrospective study of 1,171 patients with PTC from 1990 to 2012 who underwent thyroidectomy and RAI. They divided the cases into those who had $<$ or $=3$ 
GBq (75 mCi) RAI (group A) and those who had $>3 \mathrm{GBq}(75 \mathrm{mCi})$ RAI (group B). The primary outcome measure was the rate of structural recurrence. Patients were followed for a mean of 60 months. The overall rate of recurrence was $8 \%$. When corrected for TG stage, the recurrence rates were not different for $\mathrm{T} 1$ tumors (2\% group A versus $4 \%$ group $\mathrm{B} ; \mathrm{P}=.54)$ nor for $\mathrm{T} 2$ and $\mathrm{T} 3$ tumors $(\mathrm{P}=.36$ and .55 , respectively) (33).

In Elrasad, et al., (2014). study follow up 618 months after the ablative RAI-131 dose was available for 12 patients of the low-dose group all of them didn't have recurrence, and 17 cases of the high-dose group, 16 of them had no recurrence an one patient had local recurrent disease that was detected on US and WBS with RAI with re-elevation of Tg level. Obviously they could not draw a conclusion from this small number of patients, over that short duration of follow up (25).

\section{REFERNCES:}

1. Tuttle RM, Lopez N, Leboeuf $R$, Minkowitz, SM, Grewal R, Brokhin M, et al. Radioactive iodine administered for thyroid remnant ablation following recombinant human thyroid stimulating hormone preparation also has an important adjuvant therapy function. Thyroid : official journal of the American Thyroid Association.20(3):25763;2010.
On the other hand, Doi\& Woodhouse in 2007 published a meta-analysis of risk of recurrence following high- vs low-dose ablation in 2584 patients (1094 low dose and 1490 high dose) extracted from the 22 datasets, six of them were randomized controlled trials with mixed surgical status (group 2), four were cohorts with near-total thyroidectomy (group 1), and 12 were cohorts with mixed surgical status (group 3). Most studies used a high dose of (75-100 $\mathrm{mCi}$ ). The result of high-dose to lowdose group relative risk (RR) of non-ablation after the first dose was $0.58(95 \%$ CI, 0.460.74 ) for the cohort studies in group 1 and 0.88 (95\% CI, 0.78-1) for group 3. RR was 0.68 (95\% CI, 0.43-1.07) for the randomized controlled trials (group 2). The subgroup summary and individual study RRs and 95\%. Finding further support for high-dose ablation in reduction of recurrent disease (34).

\section{Bal CS, Kumar A, Pant GS. Radioiodine dose for remnant ablation in differentiated thyroid carcinoma: a randomized clinical trial in 509 patients. The Journal of clinical endocrinology and metabolism.89(4):1666-73. Epub 2004/04/09;2004.}


3. Verburg FA, de Keizer B, Lips CJ, Zelissen PM, de Klerk JM. Prognostic significance of successful ablation with radioiodine of differentiated thyroid cancer patients. Eur J Endocrinol.152(1):33-7; 2005.

\section{Taylor T, Specker B, Robbins J, Sperling} M, Ho M, Ain $K$, et al. Outcome after treatment of high-risk papillary and nonHurthle-cell follicular thyroid carcinoma. Ann Intern Med.129(8):622-7;1998.

5. Mazzaferri EL, Kloos RT. Clinical review 128: Current approaches to primary therapy for papillary and follicular thyroid cancer. J Clin Endocrinol Metab.;86(4):1447-63;2001.

6. Grewal RK, Larson SM, Pentlow CE, Pentlow KS, Gonen M, Qualey R, et al. Salivary gland side effects commonly develop several weeks after initial radioactive iodine ablation. Journal of nuclear medicine : official publication, Society of Nuclear Medicine.;50(10):1605-10; 2009.

7. Mandel SJ, Mandel L. Radioactive iodine and the salivary glands. Thyroid : official journal of the American Thyroid Association.13(3):265-71; 2003.

8. Rubino C, de Vathaire F, Dottorini ME, Hall P, Schvartz C, Couette JE, et al. Second primary malignancies in thyroid cancer patients. British journal of cancer.;89(9):1638-

44. Epub 2003/10/30;2003.

9. Sawka AM, Thabane L, Parlea L, Ibrahim-Zada I, Tsang RW, Brierley JD, et al. Second primary malignancy risk after radioactive iodine treatment for thyroid cancer: a systematic review and meta-analysis. Thyroid : official journal of the American Thyroid Association.19(5):451-7; 2009.

10. Cooper DS, Doherty GM, Haugen BR, Kloos RT, Lee SL, Mandel SJ, et al. Revised American Thyroid Association management guidelines for patients with thyroid nodules and differentiated thyroid cancer. Thyroid.;19(11):1167-214; 2009.

11. Castagna MG, Cevenini $G$, Theodoropoulou A, Maino F, Memmo S, Claudia , et al. Post-surgical thyroid ablation with low or high radioiodine activities results in similar outcomes in intermediate risk differentiated thyroid cancer patients. European journal of endocrinology / European Federation of Endocrine Societies.169(1):23$9 ; 2013$.

12. Mallick U, Harmer $C$, Yap B, Wadsley J, Clarke S, Moss L, et al. Ablation with low-dose radioiodine and thyrotropin alfa in thyroid cancer. The New England journal of medicine.366(18):1674-85; 2012. 
13. Creutzig $\boldsymbol{H}$. High or low dose radioiodine ablation of thyroid remnants? European journal of nuclear medicine.12(10):5002;1987.

\section{Johansen K, Woodhouse NJ, Odugbesan} O. Comparison of $1073 \mathrm{MBq}$ and $3700 \mathrm{MBq}$ iodine-131 in postoperative ablation of residual thyroid tissue in patients with differentiated thyroid cancer. Journal of nuclear medicine : official publication, Society of Nuclear Medicine.;32(2):252-4; 1991.

\section{Taube A, Lundell G. Prospective} randomized clinical trial to evaluate the optimal dose of 131I for remnant ablation in patients with differentiated thyroid carcinoma. Cancer.;79(1):190-1;1997.

\section{Bal C, Chandra P, Kumar A,} Dwivedi S. A randomized equivalence trial to determine the optimum dose of iodine-131 for remnant ablation in differentiated thyroid cancer. Nuclear medicine communications.33(10):103947;2012.

\section{Caglar M, Bozkurt FM, Akca CK, Vargol} SE, Bayraktar M, Ugur O, et al. Comparison of 800 and $3700 \mathrm{MBq}$ iodine-131 for the postoperative ablation of thyroid remnant in patients with low-risk differentiated thyroid cancer. Nuclear medicine communications.33(3):268-74;2012.
18. Fish SA, Basu S, Alavi A, Mandel SJ. Comparison of efficacy of $2220 \mathrm{MBq}$ versus $3700 \mathrm{MBq}$ I-131 for ablation of thyroid remnant in patients with differentiated thyroid cancer. Q J Nucl Med Mol Imaging.54(5):5603;2010.

19. Pilli T, Brianzoni E, Capoccetti F, Castagna MG, Fattori S, Poggiu A, et al. A comparison of $1850(50 \mathrm{mCi})$ and $3700 \mathrm{MBq}$ (100 mCi) 131-iodine administered doses for recombinant thyrotropin-stimulated postoperative thyroid remnant ablation in differentiated thyroid cancer. The Journal of clinical endocrinology and metabolism ;92(9):3542-6; 2007.

20. Chianelli M, Todino V, Graziano FM, Panunzi C, Pace D, Guglielmi R, et al. Lowactivity $(2.0 \mathrm{GBq} ; 54 \mathrm{mCi})$ radioiodine postsurgical remnant ablation in thyroid cancer: comparison between hormone withdrawal and use of rhTSH in low-risk patients. European journal of endocrinology / European Federation of Endocrine Societies.;160(3):431$6 ; 2009$.

\section{Kukulska A, Krajewska J, Gawkowska- Suwinska M, Puch Z, Paliczka-Cieslik E, Roskosz J, et al. Radioiodine thyroid remnant ablation in patients with differentiated thyroid carcinoma (DTC): prospective comparison of long-term outcomes of treatment with 30, 60 and $100 \mathrm{mCi}$. Thyroid research.3(1):9;2010.}


22. Schvartz C, Bonnetain F, Dabakuyo S, Gauthier M, Cueff A, Fieffe S, et al. Impact on overall survival of radioactive iodine in low-risk differentiated thyroid cancer patients. The Journal of clinical endocrinology and metabolism.;97(5):1526-35;2012.

\section{Schlumberger M, Catargi B, Borget I,} Deandreis D, Zerdoud S, Bridji B, et al. Strategies of radioiodine ablation in patients with low-risk thyroid cancer. The New England journal of medicine.366(18):1663$73 ; 2012$.

\section{Pacini F, Schlumberger $M$, Dralle $H$,} Elisei R, Smit JW, Wiersinga W. European consensus for the management of patients with differentiated thyroid carcinoma of the follicular epithelium. European journal of endocrinology / European Federation of Endocrine Societies.;154(6):787-803. Epub 2006/05/27;2006.

25. Elrasad SA-K, M .Amin, R .Abd-elhaffez, Y .Elrefaei, Sh. . Thyroid Remnant Ablation of Differentiated Thyroid Carcinoma: a comparison of Ablation success with High and Low Doses of Radioiodine. Egyptian Society of Nuclear Medicine Specialists.10(6):62$75 ; 2014$.

26. Prpic M, Dabelic N, Stanicic J, Jukic T, Milosevic M, Kusic Z. Adjuvant thyroid remnant ablation in patients with differentiated thyroid carcinoma confined to the thyroid: a comparison of ablation success with different activities of radioiodine (I-131). Annals of nuclear medicine.26(9):744-51;2012.

27. Doi SA, Woodhouse NJ. Ablation of the thyroid remnant and 131I dose in differentiated thyroid cancer. Clinical endocrinology.;52(6):765-7; 2000.

28. Pacini F, Molinaro E, Castagna MG, Lippi F, Ceccarelli C, Agate L, et al. Ablation of thyroid residues with $30 \mathrm{mCi}$ (131)I: a comparison in thyroid cancer patients prepared with recombinant human $\mathrm{TSH}$ or thyroid hormone withdrawal. The Journal of clinical endocrinology and metabolism.;87(9):4063-8; 2002.

29. Rosario PW, Mineiro Filho AF, Prates BS, Silva LC, Calsolari MR. Postoperative stimulated thyroglobulin of less than $1 \mathrm{ng} / \mathrm{ml}$ as a criterion to spare low-risk patients with papillary thyroid cancer from radioactive iodine ablation. Thyroid : official journal of the American Thyroid Association.22(11):1140-3; 2012.

30. Blumhardt R, Wolin EA, Phillips WT, Salman UA, Walker RC, Stack BC, Jr., et al. Current controversies in the initial postsurgical radioactive iodine therapy for thyroid cancer: a narrative review. Endocrine-related cancer.21(6):R473-84.;2014. 
31. Ibrahimpasic T, Nixon IJ, Palmer FL, Whitcher MM, Tuttle RM, Shaha A, et al. Undetectable thyroglobulin after total thyroidectomy in patients with low- and intermediate-risk papillary thyroid cancer--is there a need for radioactive iodine therapy? Surgery. 152(6):1096-105; 2012.

32. Tuttle RM, Sabra MM. Selective use of RAI for ablation and adjuvant therapy after total thyroidectomy for differentiated thyroid cancer: a practical approach to clinical decision making. Oral oncology.49(7):67683;2013.
33. Kruijff S, Aniss AM, Chen P, Sidhu SB, Delbridge LW, Robinson B, et al. Decreasing the dose of radioiodine for remnant ablation does not increase structural recurrence rates in papillary thyroid carcinoma. Surgery.;154(6):1337-44; 44-5 E;2013.

34. Doi SA, Woodhouse NJ, Thalib L, Onitilo A. Ablation of the thyroid remnant and I-131 dose in differentiated thyroid cancer: a meta-analysis revisited. Clinical medicine \& research.5(2):87-90; 2007. 